\title{
A Randomized Trial of Manual Phone Calls Versus Automated Text Messages for Peripheral Nerve Block Follow-Ups.
}

\author{
Gavyn Ooi \\ Thomas Jefferson University \\ Eric S. Schwenk \\ Thomas Jefferson University \\ Marc C. Torjman \\ Thomas Jefferson University \\ Kent Berg \\ Thomas Jefferson University
}

Follow this and additional works at: https://jdc.jefferson.edu/anfp

Part of the Anesthesiology Commons

Let us know how access to this document benefits you

\section{Recommended Citation}

Ooi, Gavyn; Schwenk, Eric S.; Torjman, Marc C.; and Berg, Kent, "A Randomized Trial of Manual Phone Calls Versus Automated Text Messages for Peripheral Nerve Block Follow-Ups." (2021). Department of Anesthesiology Faculty Papers. Paper 67.

https://jdc.jefferson.edu/anfp/67

This Article is brought to you for free and open access by the Jefferson Digital Commons. The Jefferson Digital Commons is a service of Thomas Jefferson University's Center for Teaching and Learning (CTL). The Commons is a showcase for Jefferson books and journals, peer-reviewed scholarly publications, unique historical collections from the University archives, and teaching tools. The Jefferson Digital Commons allows researchers and interested readers anywhere in the world to learn about and keep up to date with Jefferson scholarship. This article has been accepted for inclusion in Department of Anesthesiology Faculty Papers by an authorized administrator of the Jefferson Digital Commons. For more information, please contact: JeffersonDigitalCommons@jefferson.edu. 


\author{
A Randomized Trial of Manual Phone Calls Versus Automated Text Messages \\ for Peripheral Nerve Block Follow-Ups \\ Gavyn Ooi ${ }^{1}$ BS, MBA; Eric S. Schwenk ${ }^{2}$ MD, FASA; \\ Marc C. Torjman ${ }^{2} \mathrm{PhD}$; Kent Berg ${ }^{2 *} \mathrm{MD}$, MBA
}

\begin{abstract}
Affiliations
${ }^{1}$ Sidney Kimmel Medical College at Thomas Jefferson University, Philadelphia, PA, USA

${ }^{2}$ Thomas Jefferson University Hospital, Department of Anesthesiology, Philadelphia, PA, USA

*Corresponding author: Kent Berg MD, MBA (kent.berg@jefferson.edu)
\end{abstract}

\title{
Declarations \\ Funding
}

This study was funded by an educational grant for residents and fellows from Jeffrey Joseph, DO of the TJUH Department of Anesthesiology.

\section{Conflicts of Interest}

The authors do not declare any conflicts of interest.

\section{Ethics Approval}

Thomas Jefferson University IRB approved this study. IRB Control \#: 18D.368.

\section{Consent to Participate}

Patients were informed and consented. This consent was approved per the aforementioned IRB.

\section{Consent for Publication}

Patients were informed and consented. This consent was approved per the aforementioned IRB.

Availability of Data and Material

The power analysis and data reports used are included as supplemental material.

\section{Acknowledgements}

We thank and acknowledge Max Shilling, MD for his help in the initial abstract submitted for a poster presentation of this research and Jordan Smoker, MD, Alexander Grant, MD, and Jeffrey Mojica, MD for their invaluable help with patient recruitment. We also send special thanks to Jennifer Lessin for assistance with IRB documentation and project management. 


\section{Abstract}

2 Background and objectives: Mobile phone applications (apps) have been used for patient follow-up in the

3 postoperative period, specifically to assess for complications and patient satisfaction. Few studies have evaluated their

4 use in regional anesthesia. The objective of this study was to compare follow-up response rates using manual phone

5 calls versus an automated patient outreach (APO) app for peripheral nerve block patients. We hypothesized that the

6 response rate would be higher in the APO group.

7 Methods: A mobile app, "JeffAnesthesia," was developed, which sends notifications to patients to answer survey

8 questions in the app. We randomly assigned patients who received peripheral nerve blocks for postoperative pain to

9 either a manual phone call or an APO app group, with follow-up in each category occurring between postoperative

10 days (POD) 14-21 and 90-100.

11 Results: In total, 60 patients were assigned to the phone call group and 60 patients to the APO app group. Between

12 POD 14-21, 9 (15\%) patients were reached in the manual phone call arm, and 16 (26.7\%) patients were reached in the

13 APO arm (p=0.117). At POD 90-100, follow-up was successful with $5(8.2 \%)$ in the manual phone call group vs. 3

$14(5.0 \%)$ patients in the APO app group $(\mathrm{p}=0.300)$.

15 Conclusions: Overall response rate was poor, with comparable response rates between groups. The APO method may

16 reduce time spent by anesthesia staff on follow-up calls, but our data do not suggest this method improves response

17 rates significantly. Further studies are needed to better understand the reasons for the poor response rate and strategies

18 for improvement.

19 Keywords: anesthesiology, regional, mobile app, randomized, follow-up, automated survey 


\section{Introduction}

2 Automation of patient follow-up via mobile phone applications (apps) has potential to save time for physicians,

3 standardize responses, and increase the patient response rate. Follow-up rates as high as $60 \%$ have been reported in

4 the surgical population [1, 2]. In the specialty of anesthesiology, patients who received a peripheral nerve block have

5 been the focus of recent study. In one such study, the "Panda" smartphone application was used to assess pain after

6 peripheral nerve block in ambulatory patients [3]. Another compared response rates in an app downloaded prior to

7 surgery to a phone call in patients receiving a peripheral nerve block, and reported an app response rate of 78\% [4].

8 At Thomas Jefferson University Hospital (TJUH), anesthesiology team members routinely contact surgical patients

9 who received a peripheral nerve block to assess for complications via direct phone calls. Historically, regional

10 anesthesiology team members estimated that the rate of reaching patients by phone was $30 \%$, but the actual follow-

11 up rate was unknown.

12 Traditionally, anesthesia staff spent significant time during regular business hours calling patients for follow-up,

13 usually in the latter part of the afternoon, when clinical work was slower. Team members estimated they spent at least

14 one hour per day on follow-up efforts. Investigators suggested that the time savings provided by an automated outreach

15 process could save several hours per week and allow team members to pursue other value-generating activities instead.

16 The objective of this study was to compare follow-up response rates using manual phone calls versus an automated

17 patient outreach (APO) app for peripheral nerve block patients. The authors hypothesized that the response rate would

18 be higher in the APO group. Secondary endpoints, including patient satisfaction with nerve blocks and pain scores,

19 were also compared between the two groups. 
3 The institutional review board (IRB) approved this study (IRB Control \#:18D.368) on 6/21/2018. The trial design is

4 a randomized parallel design with two groups (manual phone calls vs. APO app) in a 1:1 allocation ratio. Inclusion

5 criteria were defined as: patients receiving regional anesthesia (nerve block single shot or catheter), $\geq 18$ years old,

6 access to a smartphone, and ability to understand spoken and written English. Exclusion criteria included: visual

7 impairment, memory/cognitive impairment, the need for English interpretive services, and prisoner status.

8 Patients who received a peripheral nerve block were enrolled at bedside by a member of the regional anesthesia team 9 on either postoperative day (POD) 1 or 2 and assigned an intervention based on a randomized sequence.

10 This randomization was produced by a computer-generated sequence with a simple balanced design, created by a 11 member of the team not involved with patient enrollment. A printed copy of the sequence was accessed by an 12 anesthesia team member each time a patient was enrolled. Study team members were not blinded in this process.

13 At the point of enrollment, patients provided verbal consent per protocol, and email addresses, cell phone numbers, 14 and date of enrollment were collected. According to group assignment, patients were then informed they would receive 15 either (1) a manual phone call to their cell phone during daytime hours of 9am-5pm or (2) receive an electronic 16 invitation to complete an electronic survey via the APO mobile app. As such, patients were not blinded to the 17 intervention they received. Patients randomized to the phone call group were told explicitly that they would receive a 18 phone call from a member of the Regional Anesthesia team, and contact information for the Anesthesia Department 19 is included in discharge paperwork for all patients. In both groups, outreach occurred between POD 14-21 and 9020100 , because these timepoints were used by the regional anesthesia team prior to this study. In addition, regional 21 anesthesia staff contact patients in the 90-100-day range because most temporary nerve injuries are resolving by that 22 time, and those rare injuries that are still present may warrant neurological consultation [5].

23 In the manual phone call group, the regional anesthesia staff member called the patient at the POD interval, specifically 24 during normal working hours from $9 \mathrm{am}-5 \mathrm{pm}$. Responses were then recorded by the team member into a research 25 database that was built in a subsection of the hospital electronic health record (EHR), but still separate from regular 26 clinical documentation. Team members spent approximately 2 months (weekly meetings, 30-45 min per meeting) with 
informatics staff to create the research database within the EHR. In the APO group, the patient's cell phone number and email address were registered into a separate HIPAA-compliant, web-based management system immediately after enrollment. The system then sent automated text messages to the patient's cell phone, inviting the patient to download the "JeffAnesthesia" app from the Google Play (Android) or App Store (iOS) and answer post-operative care surveys. Once downloaded, the patient then also received in-app reminder notifications to complete surveys at the specified outreach timepoints, as well as access to educational material promoting healthy recovery practices. Of note, research team members spent approximately 3-4 months of intermittent work (weekly meetings, 30-45 min per meeting) with the third-party vendor to develop the app and data analysis. Training the study participants in the APO group was completed as part of the study enrollment process, which usually took less than 5 min per patient.

12 The approach to sample size determination assumed that an increase in patient follow-up from the estimated $30 \%$ rate 13 to $60 \%$ with APO would potentially allow the regional anesthesia team to better understand the frequencies of rare 14 complications. The effect size was based on prior studies $[1,2]$ in which high success rates using mobile technology were reported. The sample size was determined using a power analysis with a test for equality of two proportions $(0.3$ and 0.6$)$ with alpha set at 0.05 and a power of 0.80 . With 42 subjects per treatment group $(n=84)$, there was $80 \%$ power

17 to detect a difference between the control and treatment arms. To account for dropouts and communication difficulties, a total of 60 patients per group were enrolled. Patients were then randomized into a manual phone call group or an APO app group, with a target of 60 subjects per treatment group, for a total $n=120$.

20 Patients answered the same survey questions in both arms, which were pre-determined prior to enrollment. These 21 questions allowed for the collection of data for both primary and secondary endpoints, which were analyzed at the 22 conclusion of the trial. The primary endpoint was defined as whether the patient was reached successfully via a phone 23 call in the phone call group or if the patient completed a survey in the APO app group. If a patient partially completed 24 a survey in either arm, this was counted as successful outreach for the primary endpoint. Secondary endpoints, 25 including patient satisfaction with nerve blocks and pain scores, were also measured. Patient satisfaction with nerve 26 blocks was rated $0-10$, where 0 represented very dissatisfied and 10 as very satisfied. Pain scores were also rated on a 
1 numerical rating scale where 0 represented no pain and 10 represented the worst pain imaginable. There were no

2 changes to these outcome definitions after commencement of the trial.

3 Results for 120 subjects are summarized in data tables using descriptive statistics for demographic and quantitative 4 variables for each group. Patient follow-up rates are reported as counts and percentages for each group in the analysis.

5 Statistical significance was determined using the Kruskal-Wallis test and one-way ANOVA with p<0.05 set for 6 statistical significance. Statistical analyses were performed using Systat version 13 and IBM SPSS version 25 . There

7 were no harms or unintended effects observed in either group. Data are presented as mean \pm standard deviation or 8 median (interquartile range) as appropriate. 


\section{Results}

2 A total of 120 patients who received a peripheral nerve block at TJUH were recruited into the study between 11/9/2018

3 and 1/2/2020 and randomized. The median age for the phone group was 63 (14). The median age for the APO app

4 group was 59 (12). Because the primary endpoint for this trial was defined by successfully reaching the patient, a

5 patient lost to follow-up in the specified POD interval was defined as a patient that was not reached by phone or did

6 not respond to the app. In the manual phone call arm, this definition included human error in case the team member

7 did not follow-up with the patient in the specified POD interval. The enrollment diagram is indicated in Figure 1.

8 Figure 1. Enrollment diagram. Note that "lost to follow-up" is separated into two different components for each 9 timepoint.

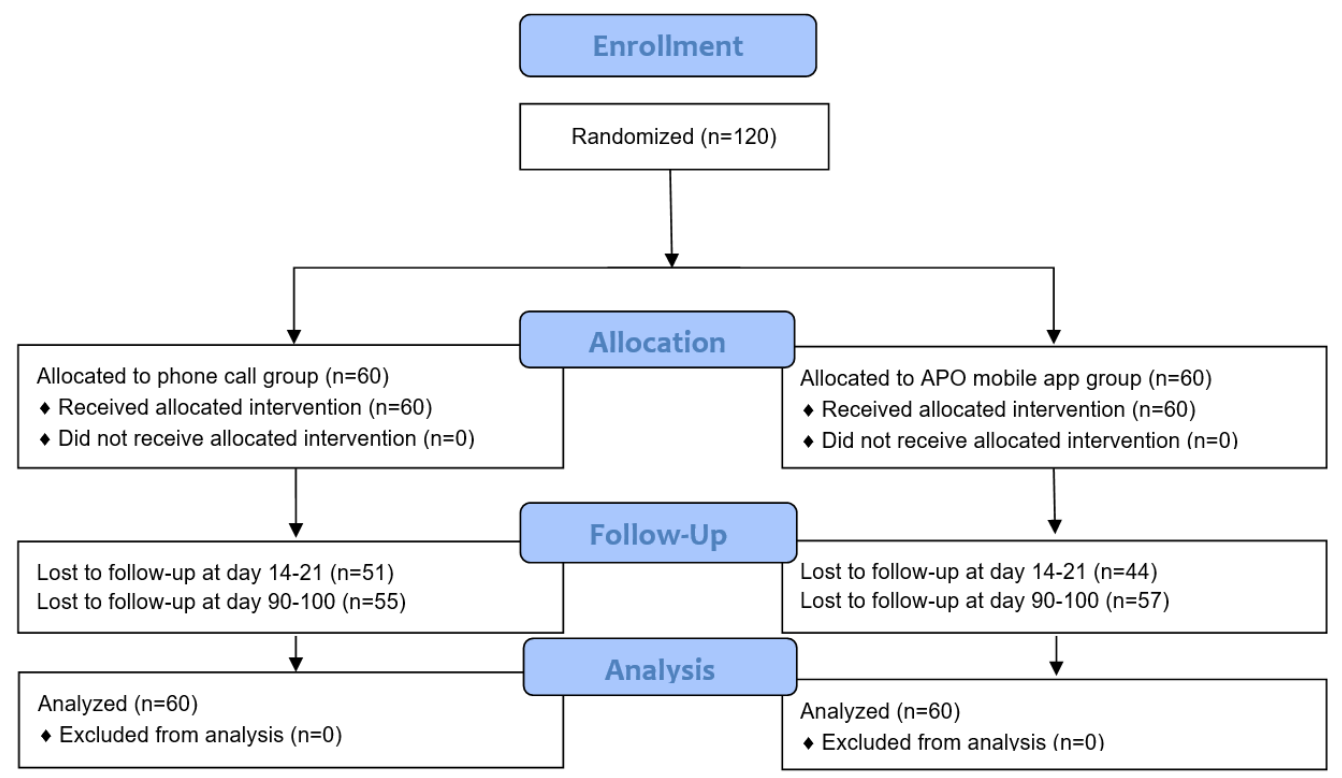

12 For the primary outcome of patients reached at POD 14-21, 9 patients (15\%) in the manual phone call group vs. 16 13 patients (27\%) in the APO app group were reached ( $\mathrm{p}=0.117)$. At this time interval, the odds ratio for reaching patients 14 with the APO app over the manual phone calls was 2.061 (95\% CI: 0.829-5.123, p=0.117). There was an absolute 15 difference of $11.67 \%$ of the APO app over manual phone calls at POD 14-21, which was not statistically significant. 16 For POD 90-100, 5 patients (8\%) in the phone group vs. 3 patients (5\%) in the APO app group were reached $(\mathrm{p}=0.300)$.

17 The odds ratio for reaching patients with the APO app over the manual phone calls was 0.474 (95\% CI: 0.113-1.989, 

$\mathrm{p}=0.300$ ). There was an absolute difference of $5.00 \%$ of manual phone calls over APO app at POD 90-100 which was also not statistically significant. Figure 2 shows an illustration of the percent of patients reached between the two interventions.

4 For the secondary outcome of pain score at POD 14-21, the mean pain score was $3.6 \pm 3.1$ in the manual phone call 5 group ( $\mathrm{n}=9)$ and $4.2 \pm 3.3$ in the APO app group $(\mathrm{n}=16)(\mathrm{p}=0.642)$. For the secondary endpoint of patient satisfaction 6 with the peripheral nerve block at POD 14-21, the mean satisfaction score was $9.7 \pm 0.7$ in the manual phone call 7 group $(n=9)$ and $8.7 \pm 2.6$ in the APO app group $(n=16)(\mathrm{p}=0.279)$. For POD 90-100, the mean pain score was $3.6 \pm$ 83.1 in the manual phone call group $(n=5)$ and $4.2 \pm 3.3$ in the APO app group $(n=3)(p=0.544)$. For patient satisfaction, 9 the mean satisfaction scores were significantly different between the two modalities: $10.0 \pm 0.0$ in the manual phone 10 call group $(\mathrm{n}=5)$ and $5.3 \pm 3.5$ in the APO app group $(\mathrm{n}=3)(\mathrm{p}=0.020)$.

11 Figure 2. Illustration of the percent of patients who were reached with the two communication modalities. There 12 were no statistically significant differences between the number of patients reached using the phone or app methods on POD 14-21 ( $\mathrm{p}=0.117)$, or on POD 90-100 $(\mathrm{p}=0.300)$.

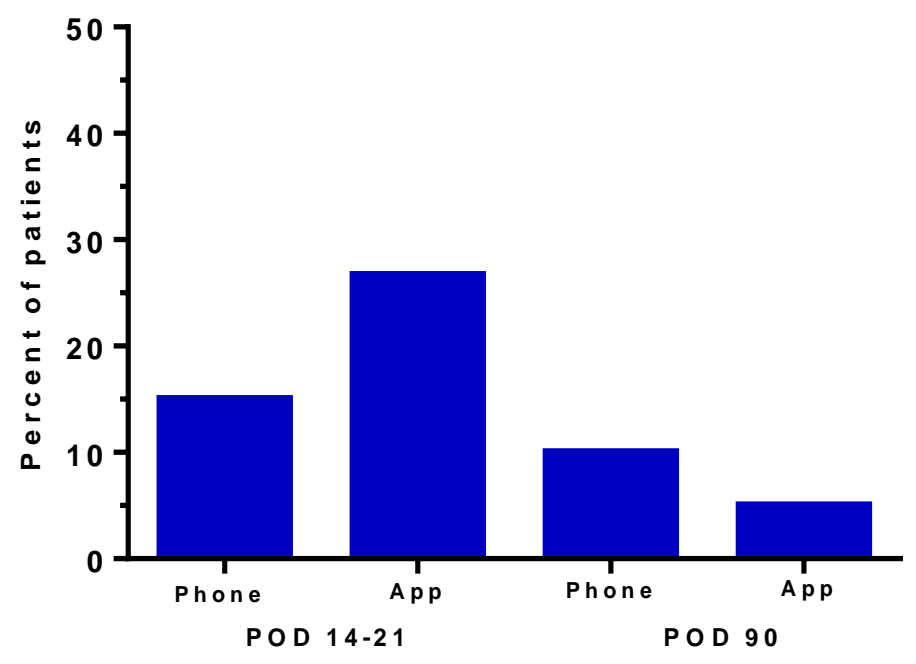

14 


\section{Discussion}

2 Between POD 14-21, a greater number and percentage of patients who received a peripheral nerve block were reached

3 via the app than those who were contacted via manual phone calls, but this difference was not statistically significant.

$4 \quad$ While most patients who experience a complication associated with a nerve block present with symptoms within the

5 first 2 weeks after surgery [6], the results of this study suggest that using an automated app yields similar results to

6 using manual phone calls in the early postoperative period.

7 The efficacy of automated or text-based messaging have been documented in settings ranging from suicide prevention

8 to medication therapy adherence [7-10]. Prior studies have shown that in the postoperative period, app-based solutions

9 have reported outreach rates greater than those observed in this study [1, 3-4]. However, patients in our study were not guaranteed to have downloaded the APO app. They were given instructions upon enrollment to download the app

11 in the postoperative period, but this download was not confirmed by the research team. In other studies, the

12 postoperative app solution was downloaded for the patient with substantial education [3-4] prior to initial outreach.

13 Therefore, we believe that our response rates reflect what occurs when hospital staff members do not download the

14 app for the patient. Taken together with the results of prior studies [1, 3-4], this implies that downloading the app is

15 the rate-limiting step. The time spent downloading the app for the patient must be accounted for if high response rates are desired. We believe our findings more closely replicate real-world practice where personnel to download apps to

17 patient phones are often not available. To maximize response rates, sufficient staff dedicated to helping patients download apps may be needed.

Although response rates did not differ between groups, the time saved by the APO app for the regional anesthesia faculty likely has important implications. Although this study did not directly measure the time required to perform

21 calls, regional anesthesia team members estimate that each patient takes on average 5 min per call, which includes

22 locating the patient contact information in the EHR, performing the phone call, and making additional attempts if

23 needed. These actions would be multiplied by 15-20 calls per day. Although daily routines vary, up to 75-100 min of

24 time could be saved per day if these calls were replaced by an APO. This could represent a large improvement in

25 efficiency for the regional anesthesia team and must weighed against the up-front cost for development and

26 implementation. Our data suggest that the APO app is no worse than traditional phone calls in terms of response rate

27 and thus the time savings may justify its use. 
Response rates at POD 90-100 were very poor with both modes of outreach, making it difficult to draw conclusions between the two methods at this time interval. However, given the time and effort needed to make these follow-up attempts, these results suggest that outreach attempts at POD 90-100 with either method do not provide substantial value and should therefore be stopped.

5 Regarding secondary outcomes of this study, there were no statistically significant differences in pain or satisfaction scores between the two outreach groups at POD 14-21, nor in pain scores between groups at POD 90-100. Interestingly, there was a difference in satisfaction at POD 90-100, with patients who were called directly reporting a significantly higher satisfaction rating. That said, given the overall low numbers of successful follow-up at POD 90100 with either method of outreach, there is likely very little clinical value in this finding.

Study Limitations

11 Selection bias was likely a limitation of this study, as participation required patients to have a smart phone and be 12 willing to receive notifications via an app/text, and therefore individuals in this study were likely more willing to 13 download an app and respond. Patients who were approached but declined participation did so largely because they did not wish to share their personal contact information or to receive automated messages from the regional anesthesia 15 team on their personal devices. As such, these results may be more applicable to those patients who are comfortable 16 with smart phone usage or to those with some basic technological literacy. Based on a 2019 survey conducted by the 17 Pew Research Center, 79\% of respondents ages 50-64 owned smart phones [11], pointing towards an increasing 18 prevalence of smart phone usage as this population ages into their Medicare-eligible years. Given this expected change 19 in market demographics, it seems prudent to migrate follow-up efforts to a mobile app platform.

20 This study may also have been limited or influenced by timing or workflow factors. At the study hospital, the regional

21 anesthesiology team members try to call patients between 9 am and $5 \mathrm{pm}$, but most typically between 3-5 pm. If 22 patients have returned to work, they may not be available for phone calls during this time or may not accept a phone 23 call from an unrecognized number. Moving to an automated app-based system allows for both continual reminders 24 and asynchronous messaging to patients, as well as an opportunity to provide pre-established educational content on 25 this platform. 
2 In conclusion, the implementation of an app-based solution for follow-up after peripheral nerve block was not superior

3 to phone calls for regional anesthesia follow up. Future studies should examine the underlying reasons why many did 4 not respond to the APO app. 
1. Semple JL, Sharpe S, Murnaghan ML, Theodoropoulos J, Metcalfe KA. (2015) Using a Mobile App for Monitoring Post-Operative Quality of Recovery of Patients at Home: A Feasibility Study. JMIR Mhealth Uhealth 2015;3(1):e18 https://doi.org/10.2196/mhealth.3929

2. Gurland B, Alves-Ferreira PC, Sobol T, Kiran RP. (2010) Using technology to improve data capture and

4. Highland KB, Tran J, Edwards H, Bedocs P, Suen J, Buckenmaier CC. (2019) Feasibility of App-Based integration of patient-reported outcomes into clinical care: pilot results in a busy colorectal unit. Dis Colon Rectum. 53(8):1168-75. https://doi.org/10.1007/DCR.0b013e3181d87468.

3. Dotto A, Dunsmuir D, Sun T, Chiu LYL, Ree R, Ansermino JM, Yarnold CH. (2020) The use of the Panda-Nerve Block pain app in single-shot peripheral nerve block patients: a feasibility study. Can J Anaesth. 67(9):11401151. https://doi.org/10.1007/s12630-020-01732-2. Postsurgical Assessment of Pain, Pain Impact, and Regional Anesthesia Effects: A Pilot Randomized Controlled Trial. Pain Med. 20(8):1592-1599. https://doi.org/10.1093/pm/pny288.

5. Yajnik M, Kou A, Mudumbai SC, Walters TL, Howard SK, Edward Kim T, Mariano ER. Peripheral nerve blocks are not associated with increased risk of perioperative peripheral nerve injury in a Veterans Affairs inpatient surgical population. (2019) Reg Anesth Pain Med. 44(1):81-85. https://doi.org/10.1136/rapm-2018-000006.

6. Fredrickson M.J., Kilfoyle D.H. (2009) Neurological complication analysis of 1000 ultrasound guided peripheral nerve blocks for elective orthopaedic surgery: a prospective study. Anaesthesia. 64(8), 836-44. https://doi.org/10.1111/j.1365-2044.2009.05938.x.

7. Perri-Moore S, Kapsandoy S, Doyon K, Hill B, Archer M, Shane-McWhorter L, Bray BE, Zeng-Treitler Q. (2016) Automated alerts and reminders targeting patients: A review of the literature. Patient Educ Couns. 99(6):953-9. https://doi.org/10.1016/j.pec.2015.12.010.

8. Berrouiguet S, Baca-García E, Brandt S, Walter M, Courtet P. (2016) Fundamentals for Future Mobile-Health (mHealth): A Systematic Review of Mobile Phone and Web-Based Text Messaging in Mental Health. J Med Internet Res. 18(6):e135. https://doi.org/10.2196/jmir.5066.

9. Berrouiguet S, Alavi Z, Vaiva G, Courtet P, Baca-García E, Vidailhet P, Gravey M, Guillodo E, Brandt S, Walter M. (2014) SIAM (Suicide intervention assisted by messages): the development of a post-acute crisis text 

0294-8.

3 10. Pop-Eleches C, Thirumurthy H, Habyarimana JP, Zivin JG, Goldstein MP, de Walque D, MacKeen L, Haberer

4 J, Kimaiyo S, Sidle J, Ngare D, Bangsberg DR. (2011) Mobile phone technologies improve adherence to 5 antiretroviral treatment in a resource-limited setting: a randomized controlled trial of text message reminders.

6 AIDS. Mar 27;25(6):825-34. https://doi.org/10.1097/QAD.0b013e32834380c1.

7 11. Pew Research Center. Mobile Fact Sheet (2019). https://www.pewresearch.org/internet/fact-sheet/mobile/. Accessed 20 March 2020. 